\title{
New Block ILU Preconditioner Scheme for Numerical Analysis of Very Large Electromagnetic Problems
}

\author{
Alex Heldring, Jaun M. Rius, and Leo Ligthart
}

\begin{abstract}
Large electromagnetic scattering and radiation problems are tackled by iterative solvers, which require the use of huge preconditioners. Most often, the incomplete LU decomposition (ILU) of the preconditioner is applied to the system matrix at each iteration. However, the preconditioner ILU cannot be done in-core when the size of the preconditioning matrix exceeds the available memory. This paper presents a new preconditioning scheme to do the preconditioner ILU in small blocks that fit in core memory. The resulting approach allows the solution of very large problems in small computers.
\end{abstract}

Index Terms-Iterative solution of linear systems, Method of Moments, numerical analysis, preconditioning.

\section{INTRODUCTION}

$\mathbf{I}$ NTEGRAL equation methods (IE) [1] are widely used in conjunction with Method of Moments (MoM) discretization [2] for the numerical analysis of electromagnetic radiation and scattering. IE-MoM formulation leads to a full linear system of order $N$, where $N$ is the number of unknowns of the problem. The operation count for the solution grows with $N^{3}$ for direct solution (LU decomposition) or with $N^{2}$ (a matrix-vector product) per iteration for an iterative method. The advent of very efficient methods for computing matrix-vector products in the iterative solution of the IE-MoM linear system has made the solution of problems involving full matrices and a very large number of unknowns (more than a hundred thousand) within the reach of present computers. Examples of such efficient algorithms are the conjugate or biconjugate methods using the fast Fourier transform [3], [4], the fast multipole algorithm [5] and its multilevel version (MLFMA) [6], or the Multilevel Matrix Decomposition Algorithm (MLMDA) [7].

However, the MoM matrices arising from large electromagnetic scattering and radiation problems are often poorly conditioned, especially when they are based on the Electric Field Integral Equation (EFIE) [1], as is the case for problems involving open surfaces (infinitely thin structures). This causes iterative methods to converge very slowly or not at all. Therefore, it is crucial to use an efficient preconditioner. A successful precon-

Manuscript received July 5, 2001; revised October 25, 2001. This work was supported by the European Comission and the Spanish Comisión Interministerial de Ciencia y Tecnología (CICYT) through Grants FEDER 2FD97-0135 and TIC 98-1037 and by the Departament d'Universitats Recerca i Societat de la Informació (DURSI) of the Generalitat de Catalunya.

A. Heldring and L. Ligthart are with the International Research Centre for Telecommunications and Radar, Delft University of Technology, 2628 CD Delft, The Netherlands.

J. M. Rius is with the Department of Signal Theory and Communications, Universitat Politècnica de Catalunya, 08034 Barcelona, Spain (e-mail: rius@tsc.upc.es).

Publisher Item Identifier S 0018-9464(02)01572-8. ditioner is obtained by creating a sparse matrix $M$ containing only the largest elements per row of the MoM matrix $A$ and multiplying by $M^{-1}$ both sides of the equation system:

$$
A \mathbf{x}=\mathbf{y} \quad \Rightarrow \quad M^{-1} A \mathbf{x}=M^{-1} \mathbf{y} .
$$

The linear system matrix, $M^{-1} A$, usually has a very good condition number and convergence can be achieved in very few iterations. The conventional preconditioning scheme computes and stores the Incomplete LU (ILU) decomposition of $M$ [8]. The preconditioner ILU is then applied at each iteration by forward and backward substitution on the working vector $A \mathrm{x}^{(\mathbf{k}}$, where $\mathbf{x}^{(\mathbf{k}}$ is the $k$ th approximation to the unknown $\mathbf{x}$.

The effectiveness of this preconditioning scheme depends strongly on the size of $M$ and on the thresholding value used in the ILU. These two parameters determine the size of the $L$ and $U$ factors (size refers here to the number of nonzero matrix elements). A problem arises when the size of the preconditioner ILU necessary to achieve good convergence is too large for the computer core memory, since the conventional ILU algorithm needs access to the entire matrices $L$ and $U$ as they are built.

This paper proposes a new block ILU algorithm, without loss of efficiency and with limited computational overhead. The blocks can be computed and stored sequentially in core memory, allowing the use of a huge preconditioner ILU that uses much more memory than is available.

\section{ALGORITHM}

The proposed blocked ILU algorithm is based on the partitioned inverse formulas [9]. If a given matrix $A$ is partitioned into four blocks

$$
A=\left[\begin{array}{ll}
P & Q \\
R & S
\end{array}\right]
$$

where $P$ and $S$ are square submatrices, then the inverse can be expressed as

$$
A^{-1}=\left[\begin{array}{ll}
\tilde{P} & \tilde{Q} \\
\tilde{R} & \tilde{S}
\end{array}\right]
$$

with

$$
\begin{aligned}
\tilde{P} & =\left(P-Q S^{-1} R\right)^{-1} \\
\tilde{Q} & =-\tilde{P}\left(Q S^{-1}\right) \\
\tilde{R} & =-\left(S^{-1} R\right) \tilde{P} \\
\tilde{S} & =S^{-1}+\left(S^{-1} R\right) \tilde{P}\left(Q S^{-1}\right)
\end{aligned}
$$

as can be verified by substituting (4) in the product $A A^{-1}$. 
In order to use (4) recursively on the preconditioning matrix $M$, it is subdivided into a chosen number of blocks of approximately equal sizes, based on the problem geometry. As a result, $M$ looks like

$M=$\begin{tabular}{|c|c|c|c|c|}
\hline$P_{n}$ & $Q_{n}^{n-1}$ & $Q_{n}^{n-2}$ & $\cdots$ & $Q_{n}^{0}$ \\
\hline$R_{n}^{n-1}$ & $P_{n-1}$ & & & $\vdots$ \\
\hline$R_{n}^{n-2}$ & & $\ddots$ & & \\
\hline$\vdots$ & & & $P_{1}$ & $Q_{1}^{0}$ \\
\hline$R_{n}^{0}$ & $\cdots$ & & $R_{1}^{0}$ & $S_{1}$ \\
\hline
\end{tabular}

where $n+1$ is the total number of blocks. Since $M$ contains the largest elements in each row of the linear system matrix $A$ and zeroes elsewhere, with proper column and row ordering the diagonal blocks $P_{m}$ and $S_{m}$ are full or almost full, while many of the off-diagonal submatrices $Q_{m}^{k}$ and $R_{m}^{k}$ are very sparse. In the following, $Q_{m}$ denotes the entire right-to-diagonal submatrix $\left[Q_{m}^{m-1} \cdots Q_{m}^{0}\right.$ ] and likewise $R_{m}$ denotes the entire below-diagonal submatrix. Furthermore, $Q_{m}^{(p)}$ denotes the block $\left[Q_{m}^{p} \cdots Q_{m}^{0}\right]$ and likewise for $R_{m}^{(p)}$.

First, the ILU factorization of $S_{1}$ is computed with a drop tolerance $\tau_{1}$. The operator $\left(U^{-1} L^{-1}\right)^{\prime}$ with $L U=S_{1}$ is represented by $\Sigma_{1}^{\prime}$, where the prime means that thresholding has been applied to drop small elements. $\Sigma_{1}^{\prime}$ is applied on the right to $Q_{1}$ and on the left to $R_{1}$, yielding the factors

$$
\begin{aligned}
& \Theta_{1}=Q_{1} \Sigma_{1}^{\prime} \\
& \Gamma_{1}=\Sigma_{1}^{\prime} R_{1} .
\end{aligned}
$$

All elements below a chosen threshold $\tau_{2}$ with respect to the largest elements in $\Theta_{1}$ and $\Gamma_{1}$ are dropped (set to zero). The resulting matrices are denoted with $\Theta_{1}^{\prime}$ and $\Gamma_{1}^{\prime}$. Subsequently, the factor

$$
\Pi_{1}=P_{1}-Q_{1} \Gamma_{1}^{\prime} \approx P_{1}-Q_{1} S_{1}^{-1} R_{1}
$$

is computed, and a dropping with threshold $\tau_{2}$ is applied to $\Pi_{1}$, yielding $\Pi_{1}^{\prime}$. Then, $\Pi_{1}^{\prime}$ is ILU factorized. The operator $U^{-1} L^{-1}$ with $L U=\Pi_{1}^{\prime}$ is represented by $\tilde{P}_{1}$ The operators

$$
\tilde{R}_{1}=-\Gamma_{1}^{\prime} \tilde{P}_{1}
$$

and

$$
\tilde{Q}_{1}=-\tilde{P}_{1} \Theta_{1}^{\prime}
$$

are not explicitly stored, but whenever they are needed as operators on a matrix or vector, (9) and (10) are invoked. This concludes step one.

Now, $\tilde{P}_{1}, \tilde{Q}_{1}$, and $\tilde{R}_{1}$ are available as operators, and so is $\Sigma_{1}^{\prime} \approx S_{1}^{-1}$. Subsequently, the operators $\Gamma_{m}^{\prime}, \Theta_{m}^{\prime}$ and $\tilde{P}_{m}$ are computed and stored sequentially for $m=2 \cdots n$. $\Theta_{m}$ is defined as $Q_{m} \Sigma_{m}$ and can be found with the recursive formula

$$
Q_{p} \Sigma_{q}^{\prime}=\left[\left[\Xi_{q-1}^{p}\right] \quad\left[-\Xi_{q-1}^{p} \Theta_{q-1}^{\prime}+Q_{p}^{(q-2)} \Sigma_{q-1}\right]\right]
$$

in which

$$
\Xi_{q-1}^{p}=-\left(Q_{p}^{q-1}-Q_{p}^{(q-2)} \Gamma_{q-1}^{\prime}\right) \tilde{P}_{q-1} .
$$

Likewise, $\Gamma_{m}$ is defined as $\Sigma_{m} R_{m}$ and can be found with the recursive formula

$$
\Sigma_{q}^{\prime} R_{p}=\left[\begin{array}{c}
\Lambda_{q-1}^{p} \\
-\Gamma_{q-1}^{\prime} \Lambda_{q-1}^{p}+\Sigma_{q-1} R_{p}^{(q-2)}
\end{array}\right]
$$

in which

$$
\Lambda_{q-1}^{p}=\tilde{P}_{q-1}\left(R_{p}^{q-1}-\Theta_{q-1}^{\prime} R_{p}^{(q-2)}\right) .
$$

Every operation in (11) and (13) is followed by a dropping with threshold $\tau_{2}$, to obtain sparser $\Gamma_{m}^{\prime}$ and $\Theta_{m}^{\prime}$ from $\Gamma_{m}$ and $\Theta_{m}$. At every level, once $\Gamma_{m}^{\prime}$ is known, $\tilde{P}_{m}$ is computed as $\tilde{P}_{m}=$ $U^{-1} L^{-1}$ with $L U=\Pi_{m}^{\prime}$, where $\Pi_{m}^{\prime}$, obtained from

$$
\Pi_{m}=P_{m}-Q_{m} \Gamma_{m}^{\prime}
$$

and a dropping with threshold $\tau_{2}$.

A close examination of (11) and (13) shows that the operators $\Sigma_{m}^{\prime}$ never have to be computed explicitly: for the computation of $\Gamma_{m}^{\prime}, \Theta_{m}^{\prime}$ and $\tilde{P}_{m}$ only $\Gamma_{k}^{\prime}, \Theta_{k}^{\prime}$ and $\tilde{P}_{k}$ with $k=$ $m-1, m-2 \cdots 1$ are needed. At each level $m$, all the previous factors are loaded and used recursively, down to level one. For symmetrical matrices like the EFIE impedance matrix, the factorization workload and storage is reduced by a factor of about one half because $\Gamma_{m}=\Theta_{m}^{T}$. The choice of the threshold values $\tau_{1}$ and $\tau_{2}$ is a tradeoff between the effectiveness and the size of the preconditioner.

Once the preconditioner factors are computed, the preconditioner has to be applied at each iteration step to a working vector $\mathrm{x}$

$$
\mathbf{y} \approx M^{-1} \mathrm{x}
$$

The vector $\mathrm{x}$ is subdivided into $n+1$ blocks corresponding to the blocks of $M$. Let $\mathrm{x}_{i}$ denote the subset of elements corresponding to block $i$ and $\mathrm{x}_{(\mathbf{i})}$ the subset of elements corresponding to blocks 1 to $i$. The same notation applies to the vector y. For block one

$$
\mathbf{y}_{1}=\Sigma_{1}^{\prime} \mathbf{x}_{1} .
$$

The following blocks are computed recursively for $m=2 \cdots n+1$ with

$$
\mathbf{y}_{(\mathbf{m})}=\left[\begin{array}{c}
\nu_{\mathbf{m}} \\
-\Gamma_{m-1}^{\prime} \nu_{\mathbf{m}}+\mathbf{y}_{(\mathbf{m}-1)}
\end{array}\right]
$$

where

$$
\nu_{\mathbf{m}}=\tilde{P}_{\mathbf{m}-1}\left(\mathbf{x}_{\mathbf{m}}-\Theta_{\mathbf{m}-1}^{\prime} \mathbf{x}_{(\mathbf{m}-1)}\right) .
$$

\section{RESULTS}

All the computations in the examples presented here were done on a PC compatible computer with a 1-GHz AMD Athlon processor and $768 \mathrm{MB}$ of RAM. The programming language was MATLAB 5 with time-critical routines coded in C. 
TABLE I

COMPARISON OF CONVENTIONAL ILU WITH BLOCK-ILU PRECONDITIONER FOR THE PEC SPHERE EXAMPLE

\begin{tabular}{c|c|c}
\hline & 1 block ILU & 32 block ILU \\
\hline ILU threshold & 0.01 & 0.01 \\
\hline matrix dropping threshold & - & 0.02 \\
\hline ILU factorisation time & $44 \mathrm{~m} .3 \mathrm{~s}$. & $55 \mathrm{~m} .14 \mathrm{~s}$. \\
\hline ILU total size & $170 \mathrm{MB}$ & $246 \mathrm{MB}$ \\
\hline ILU size in core & $170 \mathrm{MB}$ & $7.7 \mathrm{MB}$ \\
\hline GMRES step time & $1 \mathrm{~m} .10 \mathrm{~s}$. & $1 \mathrm{~m} .13 \mathrm{~s}$. \\
\hline convergence (1\% error) & $134 \mathrm{steps}$ & $98 \mathrm{steps}$ \\
\hline total computation time & $3 \mathrm{~h} .20 \mathrm{~m}$. & $2 \mathrm{~h} .54 \mathrm{~m}$. \\
\hline
\end{tabular}

\section{A. Perfectly Conducting Sphere}

As a first example, the induced surface current and bistatic RCS of a Perfectly Conducting (PEC) sphere have been calculated. A relatively small sphere was chosen ( $8 \lambda$ diameter) in order to compare the conventional ILU preconditioner with the new block ILU and test the computational overhead of the new approach. The sphere was discretized into 32768 triangular patches, with an average edge length of approximately $\lambda / 10$, leading to 49152 RWG basis functions [10]. The MLFMA near interactions matrix was used as the preconditioner matrix $M$, as suggested in [7]. The size of $M$ equaled $480 \mathrm{MB}$ and was computed in $400 \mathrm{~s}$. The resulting linear system was solved iteratively using GMRES [8], both using a conventional (one block) ILU decomposition of $M$ and using a 32-block preconditioner. The stopping criterion for the iteration was a residual relative error of less than $1 \%$.

Table I shows the timing and the matrix sizes for the two computations. The 32-block preconditioner was about $30 \%$ larger than the standard one-block preconditioner, but in the 32-block approach, the ILU operations were performed only on very small blocks that require negligible use of core memory. Since the 32-block version was larger, it was also more effective and less iterations were required. The computational overhead of the 32-block ILU versus the conventional approach was small, and was counterbalanced by the smaller number of iterations required by the new algorithm for a faster overall computation. The resulting RCS was virtually equal for the two methods and agreed well with the analytical (Mie series) solution.

\section{B. Parabolic Reflector}

The second example is the parabolic reflector used in the Transportable Atmospheric Radar (TARA) project [11] carried out at the International Research Centre for Telecommunications-Transmission and Radar (IRCTR), Faculty of Information Technology and Systems (ITS) of Delft University of Technology (DUT). The reflector diameter is $3 \mathrm{~m}$ and the focal distance is $1.54 \mathrm{~m}$. The reflector is illuminated by a dielectric rod antenna at $3.2975 \mathrm{GHz}(\lambda=9.1 \mathrm{~cm})$, radiating a linearly polarized, axisymmetric field. The measured feed radiation pattern was used in the computation. The feed is mounted in a cylindrical metal housing (diameter $17 \mathrm{~cm}$, length $20 \mathrm{~cm}$ ), supported by four thin cylindrical struts (diameter $2.5 \mathrm{~cm}$ ).

The complete antenna geometry was discretized into triangular patches. The average edge length of the triangles was 0.5
TABLE II

MoM-EFIE SOLUTION FOR THE UNSHIELDED TARA REFLECTOR USING A 20-BLOCK ILU PRECONDITIONER

\begin{tabular}{c|c}
\hline size of $\mathrm{M}$ & $220 \mathrm{MB}$ \\
\hline ILU threshold & 0.001 \\
\hline matrix dropping threshold & 0.002 \\
\hline ILU total size & $1.3 \mathrm{~GB}$ \\
\hline ILU size in core & $66 \mathrm{MB}$ \\
\hline ILU factorisation time & 4 hours $26 \mathrm{~min}$. \\
\hline GMRES step time & 5 min. $20 \mathrm{sec}$. \\
\hline convergence (1\% error) & 40 steps \\
\hline total computation time & 7 hours 59 min. \\
\hline
\end{tabular}

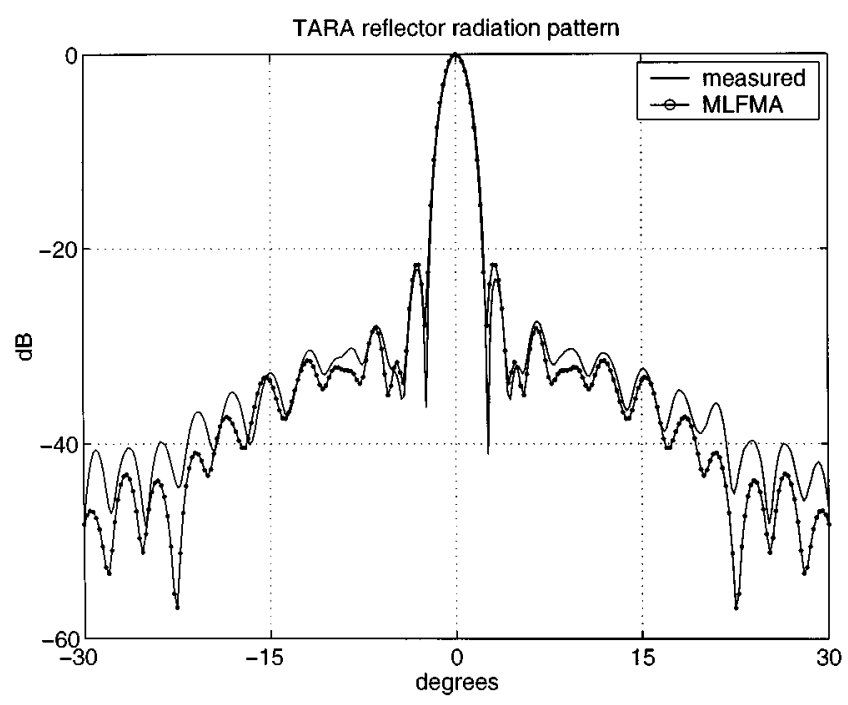

Fig. 1. Radiation pattern of the unshielded TARA parabolic reflector compared with measurements.

$\mathrm{cm}$ on the feed housing, the struts, and near the reflector edge, while on the reflector surface far from the edge was $1 \mathrm{~cm}$. This lead to 250236 triangular patches in total, and to 374348 RWG basis functions. Making use of the fourfold symmetry of the geometry, the number of unknowns was reduced to 96620 in order to compress the MLFMA near field interactions matrix [7] and the preconditioner. Unfortunately, the MLFMA far field interactions computation [7] cannot take advantage of symmetries and must deal with the whole uncompressed geometry discretization. The MLFMA near interactions matrix was built in $1000 \mathrm{~s}$ and again used as preconditioner. Computation timing and matrix sizes for the GMRES solution of this problem, using a 20-block preconditioner, are given in Table II. This problem was impossible to solve using a standard one-block ILU preconditioner: the largest one-block ILU that fitted in memory needed $480 \mathrm{MB}$ and the residual relative error stagnated at approximately $8 \%$ after 173 steps. However, the new block-ILU algorithm allowed the use of 1.3-GB ILU factors and convergence to $1 \%$ error was achieved in 40 iterations. Fig. 1 shows the reflector radiation pattern compared with measurements.

The shielded reflector configuration of TARA was also analyzed. The shield size is $2 \mathrm{~m}$ and the shield aperture angle $32^{\circ}$. The real feeder pattern was now approximated by $(1+\cos \theta)^{4.75}$. The number of RWG basis functions [10] was 1483312 . Four-fold symmetry allowed the compression of the 
TABLE III

MoM-EFIE SOLUTION FOR THE SHIELDED TARA REFLECTOR USING A 25-BLOCK ILU PRECONDITIONER

\begin{tabular}{c|c}
\hline size of M & $3.28 \mathrm{~GB}$ \\
\hline ILU total size & $3.6 \mathrm{~GB}$ \\
\hline ILU size in core & $147 \mathrm{MB}$ \\
\hline ILU factorisation time & 33 hours \\
\hline GMRES step time & $24 \mathrm{~min} .40 \mathrm{sec}$. \\
\hline convergence (1\% error) & 47 steps \\
\hline total computation time & 52 hours \\
\hline
\end{tabular}

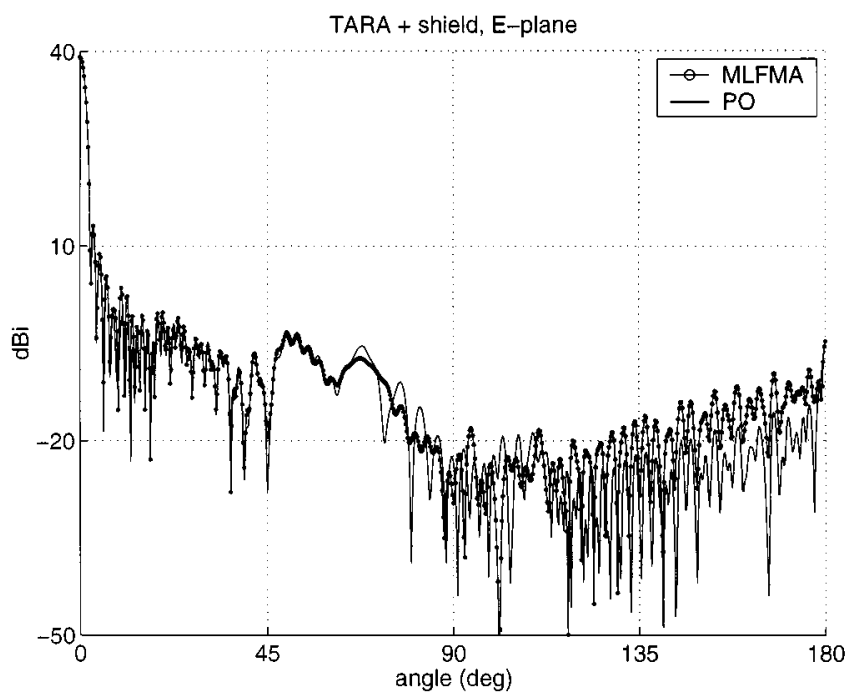

Fig. 2. E-plane radiation pattern of the shielded TARA parabolic reflector compared to $\mathrm{PO}$ approximation.

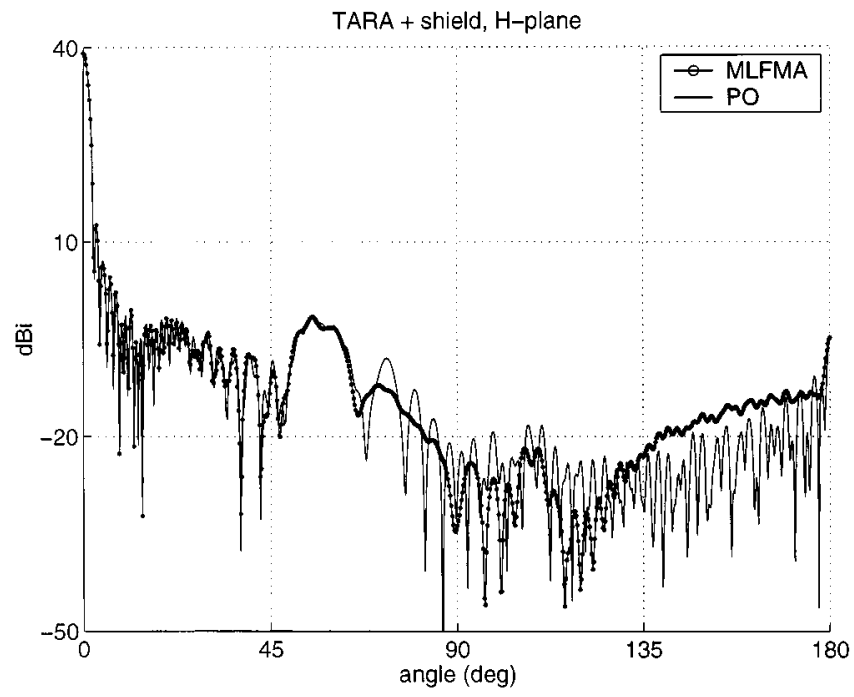

Fig. 3. H-plane radiation pattern of the shielded TARA parabolic reflector compared to $\mathrm{PO}$ approximation. near field matrix and the preconditioner to 372022 unknowns. Table III shows the computational requirements and Figs. 2 and 3 the radiation pattern computation compared to the Physical Optics (PO) approximation.

\section{CONCLUSION}

A new block-ILU preconditioning algorithm is presented that allows the use of large preconditioners on small computers, as it overcomes the requirement for a conventional ILU of not exceeding the core memory size. Larger preconditioners significantly improve the convergence speed of an iterative solution method like GMRES. The new block ILU has been applied to the MoM solution of the EFIE with small memory requirements to perform the ILU decomposition of the preconditioner blocks. The results show a small computational overhead compared to the conventional one-block ILU for relatively small problems, and excellent convergence for large problems that cannot be tackled by the conventional approach. The choice of the number of blocks is not critical; it must not be too small to allow the block-ILU decomposition in core memory, and not so large as to make the computational overhead significant.

\section{REFERENCES}

[1] N. Morita, N. Kumagai and, and J. R. Mautz, Integral Equation Methods for Electromagnetics. Norwood, MA: Artech, 1990.

[2] R. F. Harrington, Field Computation by Moment Methods. New York: MacMillan, 1968.

[3] T. Sarkar, Ed., Application of Conjugate Gradient Method to Electromagnetic and Signal Analysis. Amsterdam, The Netherlands: PIER-5, Elsevier, 1991.

[4] C. F. Wang, F. Ling, and J. M. Jin, "A fast full-wave analysis of scattering and radiation from large finite arrays of microstrip antennas," IEEE Trans. Antennas Propagat., vol. 46, pp. 409-418, Oct. 1998.

[5] R. Coifman, V. Rohklin, and S. Wandzura, "The fast multipole method for the wave equation: A pedestrian description," IEEE Antennas Propag. Mag., vol. 35, pp. 7-12, 1993.

[6] J. Song, C. C. Lu, and W. C. Chew, "Multilevel fast multipole algorithm for electromagnetic scattering by large complex objects," IEEE Trans. Antennas Propag., vol. 45, pp. 1488-1493, Oct. 1997.

[7] J. M. Rius, J. Parrón, E. Úbeda, and J. R. Mosig, "Multilevel matrix decomposition algorithm for analysis of electrically large electromagnetic problems in 3-D," Microwave Opt. Technol. Lett., vol. 22, no. 3, pp. 177-182, Aug. 1999.

[8] Y. Saad, Iterative Methods for Sparse Linear Systems. Boston, MA: PWS, 1996.

[9] W. H. Press e.a, Numerical Recipes in C, 2nd ed. Cambridge, U.K.: Cambridge Univ. Press, 1994.

[10] M. Rao, D. R. Wilton, and A. W. Glisson, "Electromagnetic scattering by surfaces of arbitrary shapes," IEEE Trans. Antennas Propag., vol. 30, pp. 409-418, May 1982.

[11] S. H. Heijnen and L. P. Ligthart, "TARA: Transportable Atmospheric RAdar," in Proc. 28th Eur. Microwave Conf., vol. 1, 1998, pp. 61-66. 